\title{
Alcoolismo e Problemas Relacionados - Dificuldades na Implementação de Estudos de Prevalência
}

\section{Prevalence of Alcoholism and Related Problems: Some Difficulties in Conducting Surveys}

\author{
Evandro da S. F. Coutinho'
}

COUTINHO, E. da S. F. Prevalence of Alcoholism and Related Problems: Some Difficulties in Conducting Surveys. Cad. Saúde Públ., Rio de Janeiro, 8 (1): 22-29, jan/mar, 1992.

This paper presents some methodological difficulties in studying the prevalence of alcoholism and alcohol abuse. It discusses the definition of alcoholic and his identification by instruments used in surveys. The advantages and disadvantages of different types of research desings for estimating the prevalence of alcoholism and alcohol-related problems are also discussed.

Keywords: Alcoholism; Prevalence; Surveys; Epidemiology

A epidemiologia parte da premissa de que os problemas de saúde não se distribuem ao acaso na população, e sim de forma diferenciada entre os diversos segmentos que a constituem. Por isso, o epidemiologista procura quantificar, através de medidas de freqüência relativa (taxas, proporções), a ocorrência das enfermidades nos grupos populacionais, assim como identificar fatores que modificam a sua maior ou menor chance (risco) de ocorrência nos diferentes subgrupos da população.

Prefere-se utilizar a expressão fator de risco no lugar de causa para se referir aos fatores que determinam a maior ou menor proporção (prevalência) de doentes num dado grupo ou subgrupo populacional. Com esta opção, procura-se substituir o conceito de causalidade presente no determinismo puro (causa necessária e suficiente) por outra que expresse a idéia de incremento ou redução na probabilidade de ocorrência da doença num conjunto de indivíduos (Rothman, 1986).

Inicialmente, a epidemiologia voltou-se para as doenças infecciosas por representarem a principal causa de morbi-mortalidade. Com a

\footnotetext{
'Departamento de Epidemiologia e Métodos Quantitativos em Saúde da Escola Nacional de Saúde Pública. Rua Leopoldo Bulhões, 1480, Rio de Janeiro, RJ, 21041, Brasil.
}

"transição epidemiológica" na virada deste século (Susser, 1985), as doenças crônico-degenerativas assumiram um papel mais relevante, paralelamente ao declínio da importância das doenças infecciosas no quadro de morbi-mortalidade da Europa e EUA. Assim, a epidemiologia passou a se ocupar também dos transtornos mentais, o que fez com que ela se defrontasse com novos problemas.

Tendo a clínica como um dos eixos que a contituíram (Almeida Filho, 1988), a epidemiologia trabalha dentro do modelo médico que tem como um de seus pressupostos a existência da doença em estudo. O que a princípio pode parecer óbvio não é tão claro nos transtornos mentais, sendo o pressuposto de existência de entidades nosológicas mais fraco na psiquiatria do que nas demais especialidades médicas (Eaton, 1986). A falta de validade dos critérios de diagnóstico nos estudos epidemiológicos dos transtornos mentais tem sido apontada como um dos empecilhos à obtenção de resultados expressivos, sobretudo no que diz respeito à etiologia. Por esta razão, a epidemiologia psiquiátrica tem priorizado a melhoria da qualidade do diagnóstico em seus estudos mais recentes (Dohorenwend \& Dohorenwend, 1982; Willians et al., 1980).

O alcoolismo talvez se constitua num dos exemplos mais significativos dessa dificuldade. 
Edwards (cit by Hill, 1985) dizia, em meados da década de 80 , não existir evidências do alcoolismo ser ou não uma doença. A ausência de um padrão homogêneo, de um curso único, ao contrário do que se pensava antes, faz com que alguns autores prefiram ver o alcoolismo como uma síndrome e não como uma doença (Hill, 1985). Entretanto, essa ainda é uma questão polêrnica.

\section{ASPECTOS METODOLÓGICOS}

Tradicionalmente, os inquéritos epidemiológicos sobre alcoolismo têm se voltado para três aspectos: a prevalência do alcoolismo e do consumo de bebidas alcoólicas, a distribuição de ambos em subgrupos populacionais e os problemas relacionados com o uso do álcool. Designaremos aqui por problemas ligados ao álcool o que se descreve na literatura do alcoolismo como alcohol-related problems, isto é, os problemas que os indivíduos que fazem uso abusivo de álcool podem ter no trabalho, na família, com a justiça.

As dificuldades que se apresentam quando essas questões são abordadas através de um enfoque epidemiológico não diferem substancialmente daquelas encontradas na epidemiologia dos demais transtornos mentais. Há dificuldades quanto ao critério diagnóstico, validade e confiabilidade da informação, preconceitos, estigmatização e ausência de informação no atestado de óbito. A seguir, algumas dessas dificuldades serão particularizadas.
1) Definição de Alcoolismo. Para lidar com doenças crônicas, endêmicas, é necessário que o investigador disponha de definições precisas e operacionais que permitam estabelecer uma clara distinção entre casos e não-casos da doença. Em psiquiatria, este pressuposto tem sido difícil de ser alcançado (Cooper \& Morgan, 1973).

Independente do delineamento do estudo, os resultados são influenciados pela definição de alcoolismo adotada. Boyd et al. (1983) compararam as taxas de prevalência de alcoolismo na ocasião do estudo (point-prevalence) e em qualquer momento da vida do entrevistado (lifetime prevalence) obtidas de uma amostra de 510 indivíduos de uma população geral, utilizando sete critérios diferentes de diagnóstico. Os dados apresentados na Tabela 1 permitem identificar uma variação destas prevalências de 1,6 a $2,4 \%$ e de 3,1 a $6,3 \%$, respectivamente. Portanto, é fundamental que os investigadores explicitem a definição adotada, para que os resultados possam ser interpretados corretamente.

O diagnóstico de alcoolismo tem sido definido com base em quatro grupos de indicadores (Schuckit, 1985): consumo, dependência psicológica, dependência física e problemas relacionados ao álcool.

Os critérios baseados no consumo consideram a quantidade, a freqüência e a variabilidade, nem sempre estabelecidas com facilidade. Sua mensuração tem sido feita, em geral, de duas maneiras:

TABELA 1. Prevalência de Alcoolismo em 510 Indivíduos de acordo com Sete Critérios de Diagnóstico

\begin{tabular}{lcc}
\hline \hline Critério de diagnóstico & $\begin{array}{c}\text { Prevaléncia no momento } \\
\text { do estudo (point-prevalence) }\end{array}$ & $\begin{array}{c}\text { Prevalência em qualquer momento } \\
\text { da vida (lifetime-prevalence) } \\
\text { \% }\end{array}$ \\
\hline RDC & 2,4 & 6,3 \\
National Council on Alcoholism & 2,4 & 6,3 \\
DSM-III abuso do álcool & 2,2 & 5,3 \\
Feighner criteria & 1,8 & 4,9 \\
CID-9 síndrome de dependência do álcool & 1,8 & 4,7 \\
DSM-III dependència do álcool & 1,6 & 3,5 \\
Alcoolismo tipo gama de Jellinek & 1,6 & 3,1 \\
\hline \hline
\end{tabular}

Fonte: Boyd et al, 1983. 
a) Identificação do padrão de ingestão de álcool através da investigação de um longo periodo (último mês, último ano);

b) Avaliação de um periodo curto, onde se estuda um certo número de ocasiões específicas ou mesmo as últimas 24 horas.

A primeira abordagem costuma priorizar a informação sobre a freqüência, tendendo a subestimar a quantidade quando comparada à segunda abordagem. Entretanto, nem sempre o consumo das últimas 24 horas pode ser tomado como indicador do consumo a longo termo. Dada a variabilidade da freqüência na ingestão de bebidas alcoólicas, esta abordagem pode levar a erros de classificação dos individuos (Poikolainen, 1987). Já se observou também que o uso de um diário para registrar o consumo de álcool costuma identificar mais bebedores sob risco do que as perguntas sobre o volume de bebida e a freqüência do seu uso (Redman et al., cit by Ryder et al., 1988).

A dependência psicológica, que se traduz num grande desconforto na ausência de álcool, é de difícil determinação dada a sua subjetividade. Já a dependência física constitui-se num critério muito restritivo, pois se caracteriza pelo aparecimento de sintomas de abstinência, os quais podem estar ausentes em indivíduos que já apresentam problemas ligados ao consumo do álcool. Por outro lado, a definição baseada nos problemas relacionados ao álcool pressupōe que o fato de alguém beber até apresentar dificuldades importantes na vida indica uma incapacidade da pessoa em intertomper o uso de bebida alcoólica.

Para Evans (1987), os dois primeiros indicadores dependem das normas culturais que prevalecem na sociedade e que estabelecem o limite entre $o$ bebedor normal $e \mathrm{o}$ bebedor excessivo ou alcoolista. Já os dois últimos se relacionam com as consequiências graves do alcoolismo.

2) Periodo do Estudo. Apesar da importância dos estudos prospectivos na identificação de mudanças nos padrões de consumo de álcool numa dada população, o custo elevado deste delineamento o torna bastante raro. Como opção de custo menos elevado, estudos transversais (inquéritos epidemiológicos ou surveys) têm sido mais freqüentes. Esse tipo de investi- gação pode ser comprometido pela modificação do consumo de bebidas alcoólicas em certas épocas do ano, principalmente pela presença de festejos e comemorações, afetando a ocorrência de problemas relacionados com o abuso de álcool (acidentes de trânsito, intoxicações agudas).

3) Instrumento de Diagnóstico. Os estudos epidemiológicos mais recentes, chamados de terceira geração (Dohorenwend \& Dohorenwend, 1982), têm considerado dois aspectos no diagnóstico psiquiátrico: a confiabilidade e a validade dos instrumentos utilizados. $O$ primeiro refere-se à capacidade de se obter os mesmos resultados em ocasiões diferentes, ou na mesma ocasião, por dois avaliadores. O segundo se relaciona com a capacidade do instrumento distinguir pessoas que são de fato diferentes quanto à característica que se está avaliando. Expressa a habilidade do instrumento em classificar corretamente os indivíduos, o que implica na adoção de uma referência ("padrão-ouro"). Portanto, para que um instrumento seja válido, é preciso que também seja confiável, mas a recíproca não é verdadeira.

Embora entre as categorias nosológicas psiquiátricas o alcoolismo seja uma das que apresente os índices de confiabilidade mais elevados na prática clínica - kappa em torno de 0,70 (Spitzer \& Fleiss, 1974), esta medida de concordância entre dois avaliadores ainda deixa muito a desejar. Em nosso meio, a concordância encontrada para os diagnósticos referentes ao abuso de álcool e outras drogas entre pacientes hospitalizados foi ainda mais baixa do que a observada por aqueles autores, com um indice kappa em torno de 0,50 (Coutinho \& Klein, 1989).

Quanto à validade, algumas diferenças têm sido descritas com relação ao tipo de instrumento, pessoa ou paciente envolvido. Segundo Poikolainen (1987), um estudo com alcoolistas em tratamento sob abstinência mostrou que o consumo de álcool referido pelos pacientes variava em mais de duas vezes, segundo o tipo de instrumento utilizado para mensurá-la. Por outro lado, a subestimação do consumo referido não é homogênea. De modo geral, os bebedores excessivos são aqueles que mais tendem a minimizar o volume de bebida ingerido. 
As informações extraídas de arquivos policiais também costumam subestimar os problemas envolvendo alcoolistas, além de serem diferenciadas segundo o estrato social destes indivíduos.

Em um estudo conduzido por Rimmer \& Chambers (1969), comparou-se a informação dada por pacientes alcoolistas sobre a presença de doença mental, inclusive alcoolismo, entre seus parentes de primeiro grau, com o diagnóstico obtido a partir de entrevistas psiquiátricas padronizadas com esses mesmos familiares. Os autores observaram que os pacientes alcoolistas subestimaram em quase três vezes a prevalência de transtornos mentais nos familiares, inclusive naqueles com história de internação psiquiátrica. Eles chamam atenção ainda para as implicações do uso desta informação sobre estudos etiológicos de alcoolismo.

Os questionários padronizados têm se mostrado superiores aos diagnósticos estabelecidos livremente na prática clínica e aos exames de laboratório. O Michigan Alcoholism Screening Test - MAST (Selzer, 1971), constituído por 25 questões, tem sido muito utilizado como instrumento de screening, sendo considerado confiável e válido. O Short MAST (com 13 itens), além de economizar tempo, perde pouco em termos de sensibilidade e especificidade (Schuckit \& Irwin, 1988). Num estudo em que foi confrontado com exames de laboratório e diagnóstico clínico não padronizado, o SMAST mostrou-se superior a estes na identificação de casos de abuso ou dependência do álcool (Cleary et al., 1988).

Schuckit \& Irwin (1988) citam, entre outros instrumentos, o teste CAGE composto por quatro perguntas. Assim como o MAST, o CAGE mostrou-se superior aos exames laboratoriais, tendo como referência ("padrão-ouro") os critérios do Diagnostic and Statistical Manual of Mental Disorders - DSM IIl (Beresford et al., 1990). Também já foi descrita uma elevada concordância entre os resultados obtidos a partir do MAST e do CAGE (Corrigan et al., 1981). Entretanto, deve-se ter claro que o resultado positivo num teste dessa natureza implica apenas numa certa probabilidade de se estar classificando corretamente os indivíduos de uma população.

\section{ESTRATÉGIAS NOS ESTUDOS DE PREVALĖNCIA}

De acordo com Schuckit (1985), deve-se ter duas regras gerais ao se discutir os diferentes delineamentos no estudo do alcoolismo:

- Nenhum estudo pode responder a todas as perguntas sobre o problema;

- Nenhum delineamento é perfeito.

Segundo este autor, a maior parte dos estudos epidemiológicos sobre o alcoolismo pertence a uma das quatro categorias gerais: informante-chave, indicador social, pacientes em tratamento e inquéritos populacionais (surveys). Os estudos latino-americanos concentram-se nas três últimas categorias (Caetano, 1984; Cardim et al., 1986; Santana \& Almeida Filho, 1987).

Antes de discutir as vantagens de cada uma dessas estratégias, é importante assinalar que a enumeração de todos os casos de uma dada doença envolve duas atividades distintas: a identificação de casos (case-identification) e a descoberta de casos (case-finding). A primeira refere-se ao processo de definição de critérios para considerar se um indivíduo é doente ou não (discriminar casos e não-casos na população), enquanto a segunda refere-se aos métodos adotados para identificar os indivíduos que preenchem os critérios que definem um caso (Cooper \& Morgan, 1973; Eaton, 1986; Willians et al., 1980).

A descoberta de casos geralmente envolve estatísticas oficiais ou estudos populacionais. $O$ método de identificação de casos nas estatísticas oficiais é o diagnóstico atribuído pelo médico atendente, com as restrições já mencionadas, enquanto nos estudos populacionais esta se dá a partir de entrevistas padronizadas. As vantagens e dificuldades inerentes às diferentes estratégias de investigação da prevalência de alcoolismo e problemas relacionados ao consumo de ảlcool são discutidas a seguir.

1) Informante-chave. Neste delineamento são identificados indivíduos capazes de informar sobre a presença de alcoolistas em seu meio. Embora seja um método muito simples e de baixo custo, a validade da informação é muito duvidosa, sendo fortemente influenciada pela 
tolerância do grupo social com relação ao consumo de bebidas alcoólicas. Por outro, lado esta estratégia é inviável em comunidades muito populosas e/ou com grande mobilidade migratória. A prevalência de alcoolismo obtida através deste procedimento costuma ser menor do que a obtida a partir dos dados de indicadores sociais e surveys (Schuckit, 1985).

2) Indicadores sociais. Embora diversas estatísticas oficiais possam ser usadas para estimar a prevalência de alcoolismo e problemas a ele relacionados, as mais freqüentes são:

- Dados sobre a venda e consumo de bebidas alcoólicas. Esta abordagem pressupõe a existência de uma correlação direta entre o consumo médio e o número de pessoas que bebem excessivamente. Ledermann (cit. by Schuckit, 1985) propõe inclusive uma fórmula para estimar o número de pessoas sob o risco de consumo excessivo de álcool a partir do volume total consumido. Dentre as críticas a este indicador, cita-se o fato de não se ter encontrado um nível elevado de correlação entre os dados obtidos deste modo e aqueles encontrados a partir de inquéritos populacionais. Sabe-se ainda que o volume da produção clandestina de bebidas alcoólicas é grande em muitos países, dentre eles o Brasil.

Mesmo onde isso não é tão grave, alguns problemas têm sido relatados. Poikolainen (1987) refere um achado intrigante quando se estuda a razão entre o consumo referido de álcool obtido a partir de inquéritos populacionais e o volume de fato consumido (cobertura ou validade agregada). Em alguns países, como a Finlândia, o volume real consumido é representado nessa razão pelo total de vendas de bebidas alcoólicas, ficando a cobertura assim calculada entre $40 \%$ e $60 \%$. Entretanto, de 1968 a 1984 estes valores caíram de $38 \%$ para $29 \%$. Tal achado não se constitui num fenômeno isolado, já que o mesmo vem ocorrendo com o tabaco. Uma explicação possível é o fato das pessoas, na sociedade contemporânea, aprenderem as respostas socialmente aceitas e que delas se espera.

- Estatísticas de mortalidade. Trata-se de um meio barato e prático de se estimar a prevalência de alcoolismo. O problema é que, nos atestados de óbito, o alcoolismo não costuma aparecer como causa básica de morte. Em decorrência disso, tem se analisado a evolução de quatro tipos de óbitos que se sabe estarem associados com o consumo excessivo de álcool: cirrose hepática, suicídio, homicídio e acidentes de trânsito.

O Detran de Porto Alegre apurou que em metade dos acidentes de trânsito havia pelo menos uma pessoa alcoolizada. Ao considerar apenas os acidentes com vítimas fatais, esta proporção subiu para $75 \%$. Em São Paulo, o estudo dos prontuários de 1136 acidentes de trânsito com vítimas, entre 1976 e 1985, revelou que $18 \%$ envolvia o uso abusivo de bebidas alcoólicas. Apesar da magnitude, acredita-se que estes números podem estar subestimados (Ministério da Saúde, 1988).

Jellinek sugeriu uma fórmula para estimar a prevalência de alcoolismo (A) a partir da proporção de mortes por cirrose hepática atribuída ao alcoolismo $(P)$, número de mortes por cirrose hepática no período específico (D), proporção de mortes por cirrose hepática entre os alcoolistas com complicações médicas (K) e a razão entre o total de alcoolistas e aqueles com complicações (R).

$$
A=(P D / K) \cdot R
$$

Segundo Cooper \& Morgan (1973), os achados a partir de inquéritos populacionais não confirmaram as prevalências estimadas a partir da fórmula de Jellinek.

Schuckit (1985) diz que as estatísticas de mortalidade, juntamente com outras fontes (ex: dados da justiça), podem contribuir para o dimensionamento dos problemas relacionados com o álcool, como as mortes violentas, suicídio, acidentes de trânsito. Nesses casos, são necessários dados de necrópsia acompanhados do exame toxicológico. Souza et al. (1986) mostraram que, no Rio de Janeiro, a proporção de óbitos por causas violentas em que houve envio de material para exame toxicológico foi de apenas $25,7 \%$ em 1973, tendo aumentado continuamente até 1984, embora não ultrapassando $54,6 \%$. Portanto, o uso desta informação em nosso meio estaria fortemente comprometida. 
3) População em tratamento. Embora esses estudos costumem ser mais caros do que os que utilizam estatísticas oficiais, eles são mais baratos do que os surveys. Nestas investigações, a taxa de prevalência é estimada dividindo-se a população atendida em decorrência do alcoolismo pela população coberta pelos serviços estudados.

Edward Jarvis, no século passado, já mostrava que as taxas de transtornos mentais assim estimadas variavam com a distância do asilo (Susser, 1985). Apesar de hoje em dia os serviços estarem mais distribuídos, outros fatores podem distorcer os dados assim obtidos.

Terris (1965) chama a atenção para o viés de seleção na admissão em hospitais psiquiátricos, que pode afetar a composição étnica e social de sua população. Por outro lado, a entrada de alcoolistas nos serviços também pode ser influenciada pelo número de vagas destinadas aos diferentes tipos de patologia, pela percepção quanto à necessidade de buscar auxílio médico para o problema, e assim por diante.

É importante assinalarmos duas dificuldades adicionais. $O$ registro de pacientes não costuma distinguir entre a primeira vez que o indivíduo recorre ao tratamento em sua vida e a primeira vez naquele serviço. Isto pode levar a duplicidade de casos no numerador da fração que estima a prevalência. Por outro lado, a regionalização da atenção médica ainda não se consolidou em nosso pais, sendo difícil identificar a qual população serve um determinado ambulatório ou hospital psiquiátrico.

As populações em tratamento em serviços médicos gerais também têm sido alvo de investigações visando estabelecer a prevalência de alcoolismo e problemas relacionados. Entretanto, a comparação da prevalência encontrada nesses grupos com aquela obtida a partir de amostras da população geral tem revelado diferenças importantes. Num estudo realizado numa região administrativa da cidade do Rio de Janeiro, a prevalência de alcoolismo foi de $8,4 \%$ na população clínica, contra $3,0 \%$ na população geral (Coutinho \& Almeida, 1989; Almeida \& Coutinho, 1990).

4) Inquéritos Populacionais ou Surveys. Trata-se do delineamento mais seguro para estimar a prevalência de doentes numa popu- lação. Além da proporção de alcoolistas, permite também conhecer a proporção de abstêmios, de bebedores de diferentes níveis e a freqüência de problemas relacionados ao álcool. Os inquéritos também possibilitam conhecer a prevalência dos diversos padrões de consumo de álcool dentro dos diferentes estratos que compõem a população, como sexo, idade, crença religiosa, nível sócio-econômico e outros, assim como mudanças neste padrão ao longo do tempo. Tem sido observado um aumento no consumo de bebidas alcoólicas em subgrupos da população que não ingeriam grandes quantidades (mulheres, idosos). Por outro lado, vem se identificando um declínio no consumo na população de países como a França, Espanha e Portugal, onde o consumo tradicionalmente era alto (Poikolainen, 1987).

Apesar das vantagens mencionadas, duas dificuldades devem ser consideradas. A primeira relativa ao elevado custo desses estudos e a segunda decorrente da ausência de certos grupos de risco potencial na amostra selecionada. É comum que o processo de amostragem localize os individuos em seu domicilio, o que deixaria de fora do estudo as pessoas que vivem nas ruas, em condiçōes precárias de vida, dentre as quais o alcoolismo pode ser um grave problema.

\section{COMENTÁRIO FINAL}

Para se estudar a prevalência de alcoolismo e dos problemas a ele associados, diferentes delineamentos de investigação têm sido adotados. Se, por um lado, alguns deles são de custo menor ou de execução mais simples, por outro lado, os seus resultados podem sofrer severas restriçōes. Esta diversidade de métodos tem levado a uma grande variabilidade nos achados (Schuckit, 1985). No entanto, mesmo a comparação de resultados obtidos através de inquéritos populacionais se torna complexa em decorrência da adoção de critérios distintos para definir alcoolismo e da escolha do instrumento de coleta de dados. Deve-se ainda considerar as diferentes fontes e composição das amostras para efeito de comparações, pois sabe-se que diversas variáveis sócio-demográficas se asso- 
ciam com a maior ou menor frequêencia de alcoolismo e problemas relacionados ao abuso de bebidas alcoólicas. Poikolainen (1987) assinala que, embora os investigadores que lidam com o alcoolismo em geral estejam bem informados sobre essas questões, elas não costumam ser consideradas nas conclusões que extraem de seus dados.

\section{AGRADECIMENTOS}

A Kátia V. Bloch pelas sugestões ao texto original.

\section{RESUMO}

COUTINHO, E. da S. F. Alcoolismo e Problemas Relacionados - Dificuldades na Implementação de Estudos de Prevalência. Cad. Saúde Públ., Rio de Janeiro, 8 (1): 22-29, jan/abr, 1992. No artigo, são apresentadas algumas dificuldades metodológicas no estudo da prevalência do alcoolismo e dos problemas relacionados com o consumo abusivo de bebidas alcoólicas. Abordam-se questões como a definição de alcoolista e os obstáculos para a sua identificação através de instrumentos utilizados em estudos populacionais. Discutem-se ainda as vantagens e desvantagens dos diferentes deliamentos de pesquisa para estimar a prevalência do alcoolismo e dos problemas associados ao álcool.

Palavras-Chave: Alcoolismo; Prevalência; Epidemiologia

\section{REFERÊNCIAS BIBLIOGRÁFICAS}

ALMEIDA FILHO, N., 1988. Epidemiologia Sem Números. Rio de Janeiro: Ed. Campus.

ALMEIDA , L. M. \& COUTINHO, E. da S. F., 1990. O Alcoolismo e o Hospital Geral. Estudo de Prevalência junto à Demanda Ambulatorial. Jornal Brasileiro de Psiquiatria, 39: 27-31.

BERESFORD, T.; BLOW, F.; HILL, E.; SINGER, K. \& LUCEY, M., 1990. Comparison of CAGE Questionnaire and Computer-Assisted Laboratory
Profiles in Screening for Covert Alcoholism. Lancet, 25: 482-485.

BOYD, J; WEISSMAN, M.; THOMPSON, W. \& MYERS, J., 1983. Different Definitions of Alcoholism, I: impact of seven definitions on prevalence rates in a community survey. American Journal of Psychiarry, 140: 1309-1313.

CAETANO, R., 1984. Manifestations of Alcohol-Related Problems in Latin America: a review. Bulletin of the Pan American Health Organization, 8: 258-280.

CARDIM, M.; ASSIS, S.; SHERZE, M.; IGUCHI, T. \& MORGADO, A., 1986. Epidemiologia Descritiva do Alcoolismo em Grupos Populacionais do Brasil. Cadernos de Saúde Pública, 2: 191-211.

CLEARY, P.; MILLER, M.; DELBANCO, T.; BUSH, B.; WARBURG, M. \& ARONSON, M., 1988. Prevalence and Recognition of Alcohol Abuse in a Primary Care Population. American Journal of Medicine, 85: 466-471.

COOPER, B. \& MORGAN, H., 1973. Epidemiological Psychiatry. Urbana: Charles C. Thomas Publisher.

CORRIGAN ; WEBB \& UNWIN, 1981. Alcohol Dependence Among General Medical Inpatients. British Journal on Addiction, 81: 237-246.

COUTINHO, E. da S. F. \& KLEIN, C. H. Fontes de Discordância em Diagnósticos Psiquiátricos em Hospitais do Rio de Janeiro, Brasil. Boletin de la Oficina Sanitaria Panamericana, 106: 396-406.

COUTINHO, E. da S. F. \& ALMEIDA, L. M., 1989. Estudo de Prevalência de Alcoolismo em uma Região Administrativa do Rio de Janeiro, Brasil. São Paulo. (Mimeo.)

DOHORENWEND, B. \& DOHORENWEND, P., 1982. Perspectives on the Past and Future of Psychiatric Epidemiology. The 1981 Rema Lapouse Lecture. American Journal of Public Health, 72: 1271-1279.

EATON, W., 1986. Sociology of Mental Disorders. New York: Praeger Publisher.

EVANS, R., 1987. Alcohol y Salud. San José: Universidad de Costa Rica.

HILL, S., 1985. The Disease Concept of Alcoholism: a review. Drug and Alcohol Dependence, 16: 193-214.

MINISTÉRIO DA SAÚDE, 1988. Programa Nacional de Controle dos Problemas Relacionados com o Consumo de Álcool-PRONAL. Brasil: Ministério da Saúde/Dinsan.

POIKOLAINEN, K., 1987. Alcohol and Health: new perspectives in survey research. In: Psychiatric 
Epidemiology (B. Cooper, ed.), pp. 407, London: Croom Helm Ltda.

RIMMER, J. \& CHAMBERS, D., 1969. Alcoholism: methodological considerations in the study of family illness. American Journal of Orthopsychiatry, 39: 760-768.

ROTHMAN, K., 1986. Modern Epidemiology. Boston: Little Brown and Company.

RYDER, D.; LENTON, S.; HARRISON, S. \& DORRICOT, J., 1988. Alcohol and Related Problems in a General Hospital and a General Practice: screening and the preventive paradox. Medical Journal of Australia, 149: 355-360.

SANTANA, V. \& ALMEIDA FILHO, N., 1987. Aspectos Epidemiológicos do Alcoolismo. In: Alcoolismo Hoje (S. P. Ramos, org.), pp. 163, Porto Alegre: Editora Artes Médicas Sul Ltda.

SCHUCKIT, M., 1985. Overview: epidemiology of alcoholism. In: Alcohol Patterns \& Problems (M. Schuckit), pp. 274, New Jersey: Rutgers University Press.

SCHUCKIT, M. \& IRWIN, M., 1971. Diagnosis of Alcoholism. Medical Clinics of North America, 72: 1133-1153.

SELZER, M., 1971. The Michigan Alcoholism Screening Test: the quest for a new instrument. American Journal of Psychiatry, 127: 89-94.

SOUZA, E.; IGUCHI, T.; SOCHATCHEVSKI, F. \& MORGADO, A., 1986. Óbitos Devidos a Drogas Ilícitas - Estudo de 12 Anos na População do Rio de Janeiro. Jornal Brasileiro de Psiquiatria, 35: 357-364.

SPITZER, R. \& FLEISS, J, 1974. A Re-Analysis of Reliability of Psychiatric Diagnosis. British Journal of Psychiatry, 125: 341-347.

SUSSER, M., 1985. Epidemiology in the United States After the World War II: the evolution of technic. Epidemiologic Reviews, 7: 147-177.

TERRIS, M., 1965. Use of Hospital Admissions in Epidemiologic Studies of Mental Disease. Archives of General Psychiatry, 12: 420-426.

WILLIAMS, P.; TARNOPOLSKI, A. \& HAND, D., 1980. Case Definition and Case Identification in Psychiatric Epidemiology. Psychological Medicine, 10: 101-114. 